\title{
Las patentes en la introducción del hormigón armado en España: caso de estudio de la Alhóndiga de Bilbao
}

\section{The patents in the introduction of reinforced concrete in Spain: "Alhóndiga de Bilbao" case study}

I. $\operatorname{Marcos}^{(*)}, \underline{\text { J. T. San José }}^{(*)}$, J. Cuadrado ${ }^{(*)}$, P. Larrinaga ${ }^{(*)}$

\section{RESUMEN}

Las estructuras de hormigón armado se introducen en España por influencia principalmente francesa. Esto sucede a finales del siglo XIX, siguiendo el esquema de funcionamiento a través de sistemas patentados imperantes en Europa. Los sistemas inicialmente cuentan con poco soporte científico, llegando a suponer incluso a principios del siglo XX un obstáculo al desarrollo de las estructuras de hormigón, tanto por la escasez de conocimiento que demuestran como por una serie de accidentes. Por ello, a partir de finales de la primera década del siglo se promulgan una serie de normativas en muchos países, que cambiarán el diseño y cálculo de las estructura de hormigón armado. Conocer el entorno del sistema de patentes, sus comienzos y las circunstancias de su aplicación permiten comprender los condicionantes de la estructuras de esa época, como se analiza con el caso de la alhóndiga de Bilbao (1906).

Palabras clave: Alhóndiga; Blanc; Hennebique; hormigón; Monier; patentes; Ribera; Zafra.

\section{ABSTRACT}

Reinforced concrete structures are introduced in Spain mainly by French influence. This happens in the late nineteenth century, following the scheme operating through proprietary systems prevailing in Europe. Systems initially have poor scientific support reaching, even early twentieth century, an impediment to the development of concrete structures, due to the lack of knowledge shown, as well as due to a series of accidents. Therefore, from the end of the first decade of the past century are enacted a series of regulations in many countries, that will change the design and calculation of reinforced concrete structure. The knowledge of the environment of the patent systems, its beginnings and the circumstances of their application allow us to understand the constraints of the early concrete structures, as presented in the Bilbao wine store (1906).

Keywords: Wine store; Blanc; Hennebique; concrete; Monier; patents; Ribera; Zafra.

(*) Universidad del País Vasco (UPV/EHU), Bilbao (España)

(**) TECNALIA, Derio (España)

Persona de contacto/Corresponding author: josetomas.sanjose@ehu.es (J. T. San José)

Cómo citar este artículo/Citation: Marcos, I., San José, J.T., Cuadrado, J., (2014). Las patentes en la introducción del hormigón armado en España: caso de estudio de la Alhóndiga de Bilbao. Informes de la Construcción, 66(534): eo24, doi: http://dx.doi. org/10.3989/ic.13.032.

Licencia/License: Salvo indicación contraria, todos los contenidos de la edición electrónica de Informes de la Construcción se distribuyen bajo una licencia de uso y distribución Creative Commons Reconocimiento no Comercial 3.o. España (cc-by-nc). 


\section{INTRODUCCION}

Actualmente existe suficiente conocimiento y normativa de aplicación para el cálculo y construcción de estructuras de hormigón armado. Sin embargo, en sus etapas iniciales se realizaron numerosas estructuras sin ningún amparo normativo y con soporte casi exclusivamente experimental, partiendo habitualmente de alguno de los diversos sistemas patentados, en los que se basó el desarrollo inicial de estas estructuras.

Las tareas de rehabilitación de edificios con estructura de hormigón armado han de realizarse desde el conocimiento histórico del edificio. Éste incluye los condicionantes originales sobre su diseño, cálculo y construcción. En este sentido, la introducción del hormigón armado en España, y la importancia que tuvieron las patentes en esa época, permite entender la lógica de aquellas construcciones.

\section{LOS ORÍGENES DEL HORMIGÓN ARMADO: SISTEMAS PATENTADOS}

El hormigón armado es un material de construcción relativamente reciente, comparado con otros como piedra, madera o tierra. Surge, en la segunda mitad del siglo XIX, de la evolución de técnicas como el tapial, en el que la tierra se compacta entre encofrados, o el hormigón en masa, impulsado por la aparición de los cementos artificiales.

La verdadera novedad del hormigón armado la constituye la asociación de acero y de hormigón en masa para configurar nuevos elementos estructurales. Edmond Coignet, precursor del hormigón en masa, emplea hormigón para rellenar el entrevigado de perfiles de acero a fin de crear un suelo resistente al fuego, en su célebre casa de Saint Denis, en 1852. Sin embargo, es Wilkinson, en Inglaterra, el que plantea en su patente de 1854, el uso de elementos metálicos embebidos en hormigón con una función resistente (1).

Pese a estos antecedentes, la invención del hormigón armado ha recaído tradicionalmente en Joseph Monier, que curiosamente no efectuó su primera patente en construcción, sino en «Agricultura, panadería y molinería» (2). Existe otra patente francesa anterior similar, correspondiente a Lambot, autor de una barca imputrescible construida con cemento y alambre, que presentó en la Exposición Universal de París de 1855 y que al poco cayó en el olvido (3).

En sus primeras etapas, el hormigón armado se encuentra muy vinculado a la creación de sistemas constructivos, cuyos inventos se basan más en intuiciones, con un tremendo sentido práctico, que en desarrollos científicos. Es en consecuencia un conocimiento totalmente experimental. Bajo la doble perspectiva de la invención y de la naturaleza de quienes impulsaban nuevos sistemas constructivos, en su mayoría industriales (Monier, Wilkinson, Coignet, etc.), adquiere relevancia la protección de los sistemas inventados.

El nacimiento del sistema de patentes puede situarse en Francia con la patente de Monier. A partir de entonces se suceden diversos sistemas, algunos de los cuales además tienen un notable éxito en el mercado y que son explotados a nivel internacional. En la última década del siglo XIX se produce en Francia un elevado registro de patentes. Basta indicar los sistemas de Hennebique, Coignet, Cottancin, Consideré,
Bordenave, entre otros (Figura 1). Además, la exposición universal de París de 1900 supuso todo un hito y una demostración mundial sobre las posibilidades de construcción de este nuevo material y una muestra al mundo de la capacidad de la industria francesa.
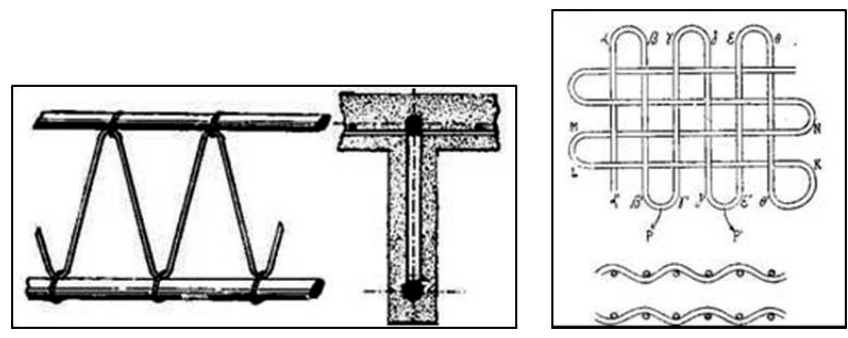

Figura 1. Sistemas Coignet y Cottacin.

El mercado centroeuropeo sigue sus propias dinámicas, y aunque las patentes francesas tienen presencia en centro, este y norte de Europa, estos países se ven más influenciados por el desarrollo alemán, cuyo grado de penetración es mayor (4). En Rusia, por ejemplo, pese a que Monier patenta su sistema en 1880, no llega a ser explotado. Sin embargo, su sistema se populariza años más tarde, de la mano de July Hook \& Co, que lo comercializa a través de Wayss (5).

En definitiva, puede afirmarse cómo el hormigón armado cuenta en Europa con dos grandes focos: Francia y Alemania. El primero de ellos extiende su influencia sobre los países occidentales como España, Inglaterra, Bélgica, Suiza, Italia o Portugal, donde sus sistemas, siempre en competencia con otros locales e incluso estadounidenses, se disputan el mercado. El modelo más elevado de estrategias de penetración queda representado por la casa Hennebique, que publicita las bondades del muevo material estructural a través de las experiencias propias y una intensa labor de difusión comercial, sin prestar excesiva atención a fundamentos científicos. Sin embargo, el modelo alemán basa su expansión, no solo en estrategias comerciales, sino en investigar y difundir los conocimientos científicos y los ensayos que se realizan para ello. De esta manera, la firma de Wayss impulsa de una manera definitiva el conocimiento y difusión del nuevo material estructural.

\section{LAS PRIMERAS ETAPAS DEL HORMIGÓN ARMADO EN ESPAÑA}

\subsection{La introducción del hormigón armado en España}

En la última década del siglo XIX en Europa y en Estados Unidos existían ya un número relevante de obras construidas en hormigón armado. Sin embargo, en España su introducción tiene lugar a finales del siglo XIX bajo influencia francesa. El primer caso conocido en cuanto a su empleo estructural es un depósito de agua en Puigverd (Lleida) construido en 1893 por Francesc Macià, según el sistema Monier.

Pese a este inicio de actividad, la aplicación de hormigón armado seguía siendo bastante limitada y no tuvo unos comienzos claros hasta los últimos años del siglo XIX, consolidándose como una alternativa estructural en la primera década del siglo XX, siempre asociado a virtudes tales como la durabilidad, el comportamiento al fuego y, por supuesto, 
su rentabilidad económica. Todas estas cuestiones, y muchas más, quedan extensamente recogidas en el magnífico trabajo de tesis doctoral llevado a cabo por el profesor Antonio Burgos Núñez (6) que presenta el estado del conocimiento de la arquitectura y la ingeniería civil en Español, desde el 1998 hasta la Guerra Civil, describiendo con todo lujo de detalles a todos y cada uno de los precursores del hormigón en España.

Los primeros edificios con estructura íntegramente construida en hormigón armado corresponden a sendas fábricas de harina: Ayala en Badajoz y La Ceres en Bilbao (7). Ambas son prácticamente coetáneas, la primera y quizás la más antigua, de Ribera y la segunda de Hennebique, en el año 1900. Ambas se construyen con hormigón para evitar los riesgos de la destrucción de las instalaciones por incendio y evitar sucesos como los de la fábrica de Caramujo (Portugal), originaria de 1889, devastada por un incendio y reconstruida entre $1897 \mathrm{y}$ 1898 empleando el sistema Hennebique.

La primera de las más de 150 patentes que se registraron en España relacionadas con el hormigón armado corresponde al sistema Monier (1884). La mayor parte de ellas se centran en sistemas constructivos y estructurales de edificaciones y obra civil, si bien abundan las relativas a traviesas de ferrocarril, tuberías o depósitos (8).

Se han seleccionado varios sistemas de patentes en función de la influencia que tuvieron en España. Los sistemas analizados con mayor detalle son los correspondientes a Monier, por ser el pionero, Hennebique, Blanc, Ribera y Zafra. De ellos solamente, los sistemas Monier y Hennebique son ampliamente conocidos a nivel internacional.

\subsection{Las patentes extranjeras}

Siguiendo el orden cronológico, la primera patente de hormigón armado en España corresponde a Monier (9), en 1884, contando con adición posterior en 1886 (10). Su explotación corrió inicialmente a cargo de Francesc Macià, quedando posteriormente a cargo del arquitecto barcelonés Claudio Durán. $\mathrm{Su}$ sistema contempla todo tipo de construcciones: traviesas de ferrocarril, vigas y viguetas para pisos o puentes, depósitos, tuberías, pavimentos, etc., todas ellas basadas en un «armazón de hierro» en forma de rejilla y con cualquier sección transversal. Posteriormente se «baña con cemento» para proteger el hierro y para dotar al elemento de la resistencia necesaria.

La explicación relativa a las vigas es escueta, no ocupando más de un par de párrafos y tres croquis. Inicialmente están destinadas a puentes y puentecillos. En suelos de edificación, recurre a viguetas metálicas en doble $\mathrm{T}$, y rellena el entrevigado con hormigón combinado con diferentes tipos de armado, no demostrando en absoluto un gran conocimiento estructural. En la adición antes referida de Monier, se efectúa una descripción más completa del proceso de fabricación de los armazones de hierro y describe la maquinaria empleada para ello. Además, contempla la posibilidad de la colocación de cemento o mortero colado, en vez de aplicarlo directamente sobre el armazón. Incluye una descripción mucho más detallada de muros y ejecuciones más versátiles para techos abovedados y suelos, si bien en estos últimos mantiene las viguetas en doble $\mathrm{T}$.

La siguiente patente relevante es la de Hennebique, en 1892, (11) a la que seguirán otras ocho más de este autor. Tratan todas ellas diversas soluciones para tuberías, vigas y viguetas (Figura 2), muros, sistemas estructurales generales y traviesas de ferrocarril. En su patente de 1898, Hennebique aporta el clásico armado del sistema, consistente en dos o más barras inferiores en la sección de la viga, ascendiendo una de ellas hacia la parte superior a medida que se aproxima a los apoyos (12), definiendo claramente lo que será su sistema habitual de armado. Se expone la posibilidad de realizar vigas continuas en la propia patente, así como la existencia de estribos en forma de U, de hierro laminado, y anclados en la cabeza de la viga. Estos estribos se disponen con mayor frecuencia en las proximidades de los apoyos, apreciándose la evolución hacia lo que será su sistema habitual y los esquemas de armado empleados a finales del XIX y al comienzo del XX. En lo referente a pilares, afirma que es extrapolable el sistema, si bien no da indicación alguna sobre su ejecutoria. Sin embargo, a través de la difusión en revistas y literatura especializada, la firma sí exponía la tipología básica del armado de pilares, consistente en cuatro barras longitudinales unidas por unos estribos formados por chapas planas caladas en dichas barras.
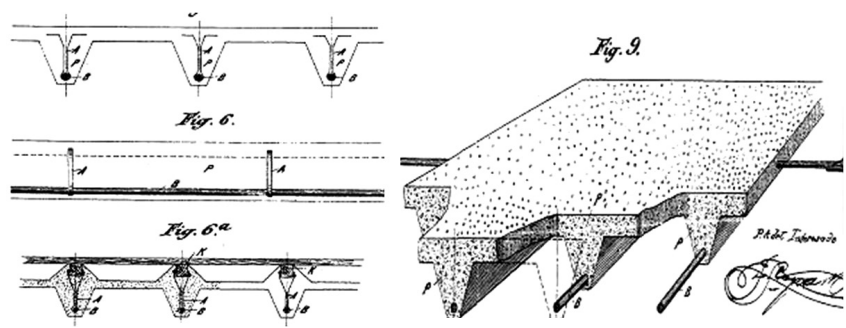

Figura 2. Viguetas Hennebique. Fuente: Patente $n^{0} 13652$ (1892).

La firma se instaló en 1898 en España, adoptando la misma sistemática de trabajo ya implantada en Francia y otros países europeos, mediante un agente general y concesiones territoriales. Estos concesionarios debían pagar una suma fija para tener derecho a la explotación de la patente, debiendo además garantizar un mínimo de obra sujeta a patente. Adicionalmente, debían abonar a la casa Hennebique un $10 \%$ de los ingresos de la parte de obra sujeta a dicha patente (13). De esta forma, los concesionarios se convertían en una fuerza comercial notable, al tratarse de constructoras implantadas en cada territorio con presencia comercial propia. A cambio, recibían el derecho a usar la patente del sistema con el desarrollo de planos y proyecto para los concursos por parte de la casa matriz, bien desde Madrid, donde estaba el agente general, o bien desde la central parisina. Correspondía al agente general la defensa de la patente, incluso ante los tribunales, y la supervisión del buen empleo del sistema en la obra.

Examinados algunos ejemplos desarrollados por la firma (14), para el cálculo de la armadura en flexión, se establece arbitrariamente el reparto del momento flector a partes iguales entre la cabeza comprimida y las armaduras traccionadas. Se considera que los forjados contribuyen en la compresión del hormigón, y se emplean para determinar la posición de la fibra neutra atendiendo a la máxima tensión admisible del hormigón, uniformemente distribuida en la cabeza comprimida (2,45 MPa). Posteriormente se calcula al área de acero en el equilibrio de la otra mitad de momento flector, admitiendo una tensión de 98,1 MPa.

En 1901 se registra la patente del sistema Blanc, a cargo de Joseph Blanc, ingeniero francés. Dicha patente es el soporte 
técnico de la Compañía de Sestao, que protagonizó una gran pugna comercial con la compañía de Ribera y con los concesionarios de Hennebique. Así, la primera gran obra que ejecuta la Compañía de Sestao es la nueva Alhóndiga de Bilbao, un edificio de cuatro plantas que ocupa una manzana en el ensanche bilbaíno, ganando el concurso a la empresa de Ribera y al concesionario local de Hennebique.

En el sistema de Blanc, se destaca la consideración de la viga junto con el forjado como sección resistente, que funciona monolíticamente. Al igual que con el resto de técnicos, realmente se producía un cálculo y un acomodo del sistema inicialmente patentado a cada obra con criterios más detallados, en función del conocimiento y experiencia de sus técnicos. Analizados los criterios de cálculo, se comprueba como considera que los flectores se equilibran por mitades entre hormigón a compresión y acero a tracción, quedando la fibra neutra en la mitad del canto; para vigas en T se encuentra a 2/3 de la distancia entre el centro de las compresiones y el de las tracciones. En cuanto a la estimación de la solicitación en la viga, se establece $\mathrm{M}=\mathrm{PL}^{2} / 12$ para vigas bi-empotradas, $\mathrm{M}=\mathrm{PL}^{2} / 8$ para vigas bi-apoyadas y $\mathrm{M}=\mathrm{PL}^{2} / 10$ para vigas maestras. Las tensiones máximas admisibles consideradas son de 2,45 $\mathrm{MPa}$ para el hormigón a compresión (2,94 MPa en pilares), 98,1 MPa para el hierro pudelado (hierro colado removido/pudelado en horno reverbero), altamente descarburado se asimila a la ferrita con elevadas inclusiones no metálicas, y, por otro lado, acero (vía Bessemer) de 117,7 MPa.

La novedad que aporta el sistema Blanc es la del comportamiento de la sección transversal en forma de T, con acartelamiento en la entrega del forjado sobre la viga, lo cual le proporciona una gran capacidad resistente y un gran monolitismo, que logra enlazando las armaduras transversales de la viga con las del forjado. Esta consideración publicitada por la empresa no tiene nada de novedoso, ya que el resto de competidores también adoptan esa solución, si bien solamente la exponen en sus documentos de cálculo, reservados a un público de carácter técnico. La Compañía de Sestao afirma que su sistema está sustentado en la experiencia y alejado de explicaciones científicas (15).

\subsection{Ribera y Zafra}

Siguiendo la evolución del hormigón armado en el extranjero y la avalancha de patentes y sistemas, especialmente franceses, los técnicos españoles establecieron contacto con el nuevo material. Además de los mencionados Maciá y Durán, otros muchos se acercaron al nuevo material. Entre todos ellos, destacan las figuras de Ribera y Zafra.

José Eugenio Ribera, Ingeniero de Caminos, es considerado como el precursor del hormigón armado en España. Su importancia no radica en la naturaleza de sus patentes, sino en su capacidad a la hora de abordar proyectos y ejecuciones de obra, así como de asegurarse una difusión técnica y comercial de las mismas. Ribera inicialmente fue representante de la firma Hennebique, con la que realizó sus primeros trabajos: el tablero de un puente en Ciaño, en 1897, los forjados de la cárcel de Oviedo y el depósito de Llanes (16) o el de 1906 en Sabadell (17). No obstante, pese a declararse «decidido partidario» del sistema Hennebique, considera que no es la mejor solución en todos los casos, motivo por el que declara haber adjudicado obras a Durán, «mi colega y competidor», que explotaba el sistema Monier. Pero va más allá, y afirma haber empleado no solo los sistemas Hennebique y Monier, sino también los Coignet, Dubois y Boussiron, a los que también añade disposiciones personales basadas en su conocimiento y experiencia (18), tal y como se aprecia en la Figura 3.

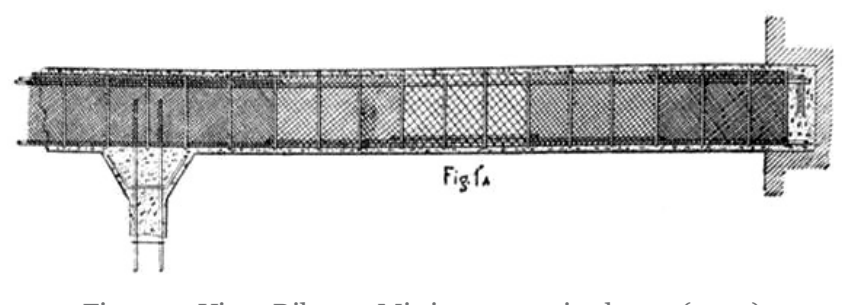

Figura 3. Vigas Ribera «Mi sistema y mis obras» (1902).

Entre las críticas que Ribera hace a los sistemas entonces vigentes, se encuentra la del elevado costo económico de los derechos de patente, que estima que puede alcanzar hasta un $20 \%$ de la obra. Por ello, se decanta por emplear el sistema más acorde con las necesidades y la economía de cada obra en particular (19). Se trata en suma, de un Ingeniero que abandona el servicio al estado y se convierte en proyectista y constructor, fundando la Compañía de Construcciones Hidráulicas y Civiles. Su planteamiento es el de la búsqueda de la solución óptima en plazo y precio, factores ambos críticos para lograr la adjudicación, sin ser determinante el sistema empleado.

Pese a esta visión sobre el hormigón armado, Ribera patenta un sistema de vigas y pilares en Julio de 1901, con una adición en el mismo año (20). Las vigas constan de armadura inferior (posibilidad de varias armaduras en la adición a la patente), una armadura superior de menor sección y una envolvente de tejido metálico o de metal deployé, junto con una serie de redondos a modo de horquilla que dan estabilidad al conjunto del armado y mantienen el tejido en su posición durante el hormigonado. Los pilares que contempla tienen igualmente el tejido envolviendo las barras de acero longitudinales.

Juan Manuel de Zafra era conocido como un brillante ingeniero con realizaciones en hormigón armado en la primera década del nuevo siglo, especialmente en el mundo de los puentes de ferrocarril y carreteras (21). Pese a ser la persona que comenzó con la docencia del Hormigón Armado en la Escuela de Caminos en 1910, y adoptar en esta época posiciones públicas contrarias al sistema de patentes, registró cuatro patentes en 1902, en el ámbito del uso estructural del hormigón armado.

Sus patentes abarcan con detalle soluciones abovedadas para pisos, armados de vigas (Figura 4), conjunto de vigas y forjados para suelos formando secciones en $\mathrm{T}$ y armadura transversal para pilares, postes y similares. Todas ellas adoptan una estructura mucho más ordenada y razonada en su exposición aportando, a diferencia de las citadas anteriormente, datos concretos sobre longitudes de barras de acero o relaciones de diámetros entre ellas. La lectura de las patentes pone de manifiesto el profundo conocimiento que demuestra para su época, abordando por ejemplo los "planos de rotura" inclinados en pilares, que son armados a través de una serie de celosías triangulares que atan entre sí sus armaduras longitudinales (22).

Zafra aplica criterios científicos y técnicos en sus clases y apuntes, mucho más allá de los esquemas de funcionamiento, 
únicamente empíricos, que preconizan las diversas patentes. Esta mentalidad explica sin duda la singularidad adoptada en la redacción de sus patentes, que no son una referencia de estos sistemas, más allá del propio uso que Zafra hiciera de ellas en sus obras.

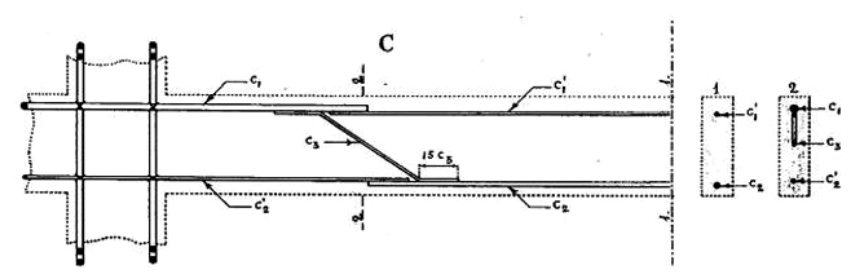

Figura 4. Armadura vigas sistema Zafra, Fuente: Patente española 29864 (1902).

\section{LA DECADENCIA DEL SISTEMA DE PATENTES}

A la imagen de falta de trasparencia y de conocimiento en el hormigón armado contribuyeron en buena medida una serie de accidentes, que se instrumentalizaron para poner en entredicho la seguridad de las estructuras erigidas en hormigón armado. El primer accidente relevante corresponde a una pasarela en la exposición de París de 1900 (23), construida con el sistema Matrai, seguido por el hundimiento del Hotel Zum Bären en Basilea en 1901, basado en el sistema Hennebique (24). En España también hubo un accidente notable en 1905, el del tercer depósito en Madrid a cargo de la empresa de Ribera, con el resultado de 30 fallecidos (25). Posteriormente hubo otros colapsos relevantes en Berna (1906) y en Milán (1908) (26).

Fruto de las numerosas obras que se van sucediendo en Francia y en el ánimo de ordenar el panorama técnico, se aprueba en 1900 la comisión encargada de establecer procedimientos de normalización y aprobación de estructuras de hormigón armado. Ésta lleva a cabo los ensayos sobre algunos edificios de la exposición de 1900, establece las reglas de cálculo y de comportamiento de los materiales y desarrolla una campaña experimental cuyos resultados complementan el conocimiento existente.

Todo ello da lugar a la Circular francesa de 1906 (27), que viene a responder a la necesidad de contar con una regulación para el proyecto y la ejecución de estructuras de hormigón armado con unos mínimos requisitos de seguridad. El fenómeno es común a otros países, y se publican, entre otras, normas suizas (1903 y 1910), alemanas (1904 y 1907), estadounidenses (1908 y 1910), italiana (1907) o rusas (1908 y 1911). En España no se aprobó ninguna norma para el proyecto y el cálculo de estructuras de hormigón armado hasta la Instrucción de 1939, por lo que fue frecuente la adopción de la Circular francesa en las primeras décadas del siglo.

El desarrollo normativo va parejo no solo al desarrollo científico, sino a la formación con la que cuentan los ingenieros dedicados al cálculo de estructuras. En Francia se inaugura la docencia del hormigón en 1897-98, en la Escuela Nacional de Puentes y Caminos. En el curso 1910-11 estas enseñanzas comienzan en la Escuela de Caminos de la mano de Zafra, quien asume públicamente en España la defensa de un nuevo estatus del hormigón basado en el conocimiento y la justificación científica frente al sistema de patentes, manteniendo una agria polémica con Hennebique a través de la Revista de Obras Públicas, en varios de sus números.

La conjunción de factores económicos, técnicos, docentes y normativos es, en consecuencia, lo que produce la desaparición paulatina del sistema de patentes, que sobrevive hasta la segunda década del siglo XX. Caso de fijar un hito histórico en España para el inicio del declive, este sería la aparición de la Circular francesa de 1906, aunque esta normativa y el conocimiento que lleva implícito, aún tardarán en calar en la comunidad técnica y en el sector constructor.

Curiosamente la Compañía Anónima del Hormigón Armado de Sestao (Vizcaya), una de las grandes competidoras de Ribera, comienza su primera gran obra en 1906, la Alhóndiga Central de Bilbao, y tiene una importante actividad en los siguientes años. En esta edificación no se aplica en absoluto la nueva normativa. Es más, ni siquiera se tienen en cuenta los criterios de cálculo publicitados por la firma (28). Aún así, el sistema todavía pervive de la mano de los industriales más potentes, ya que sus empresas cuentan con excelentes técnicos y están habituados a trabajar con el material.

\section{CASO DE ESTUDIO: LA ALHÓNDIGA CENTRAL DE BILBAO}

La que fue Alhóndiga municipal de Bilbao, denominada en su época nueva Alhóndiga o Alhóndiga Central, era un edificio concebido para sustituir y unificar los cinco locales destinados al comercio fundamentalmente de vinos, licores $\mathrm{y}$ aceites.

En lo referente a la construcción de su estructura, se presentaron tres contratistas, resultando adjudicataria de la obra «La compañía anónima del hormigón armado de Sestao", los detalles de las empresas licitadoras se adjuntan en la Tabla 1, según consta en el Archivo histórico de la Diputación Foral de Bizkaia (Signatura Bilbao Hacienda 0037/002). Las obras comenzaron en 1906, entrando en servicio en 1909.

La ejecución y el proyecto corrieron a cargo de la Compañía de Sestao, que aportó planos y cálculos relativos a la estructura (Figura 5), conservándose algunos planos de la estructura adjudicada, aunque muy degradados. En ellos, se puede comprobar algunos criterios geométricos y de armado, correspondiendo los esquemas de las vigas a los de la patente, aunque no presentan el acartelamiento de la losa en el encuentro con la viga, ni en proyecto ni en la realidad.

Tabla 1. Cuadro resumen licitación obras de La Alhóndiga de Bilbao.

\begin{tabular}{|l|c|l|l|}
\hline Empresa licitadora & Importe oferta (ptas) & Sistema patentado & Observaciones \\
\hline Compañía de Construcciones Hidraúlicas y Civiles & $872.453,84$ & Ribera & - \\
\hline Lostaló, Arrizabalaga y Compañía & $899.741,10$ & Hennebique & \multirow{2}{*}{ Incluyen planos y cálculos } \\
\hline Compañía Anónima del Hormigón Armado de Sestao & $840.000,00$ & Blanc & \\
\hline
\end{tabular}


Con motivo de las obras, y para analizar el estado de la estructura, se llevaron a cabo en 2001 diversos trabajos de indagación por parte de LABEIN (actualmente Tecnalia) en el edificio, reflejándose someramente parte del contenido en el presente artículo. Básicamente se realizó una inspección visual del estado general de estructura y fachada, un análisis de las propiedades del acero y del hormigón, el estudio de la durabilidad de la estructura y, en la medida de lo posible, un análisis de la morfología interna de la estructura y su relación con los cerramientos. Este último trabajo resultó de suma importancia para conocer los criterios reales de armado en el edificio, facilitados por los trabajos de vaciado interno.
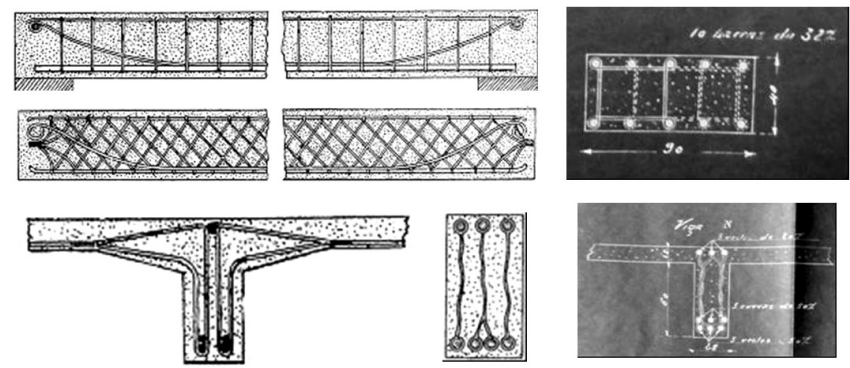

Figura 5. Armados según patente Blanc (izq.) y según proyecto alhóndiga (dcha.). Fuente: Compañía Anónima del Hormigón Armado Sestao.

Siempre existen barras inferiores de mayor diámetro, de las cuales el 50\% asciende hacia los apoyos (Figura 6), aproximadamente a un tercio de la luz, y una superior de montaje; entre ellas se disponía el entramado del estribado. Se confirma la inexistencia de armado específico frente a momentos negativos en el apoyo sobre los pilares, más allá de las barras que ascienden desde el centro de vano. Las armaduras de las vigas finalizan en el nudo, no existiendo continuidad entre los diferentes vanos. Además, los extremos de las barras están doblados en forma de "ganchos» de reducido radio. Por otra parte, se pudo comprobar cómo la armadura de la losa no tenía ninguna vinculación con el estribado de la viga, que sí es aproximadamente fiel a la patente. En cuanto a los pilares, lo representado en las secciones transversales de los planos se corresponde con la tipología detectada in situ.

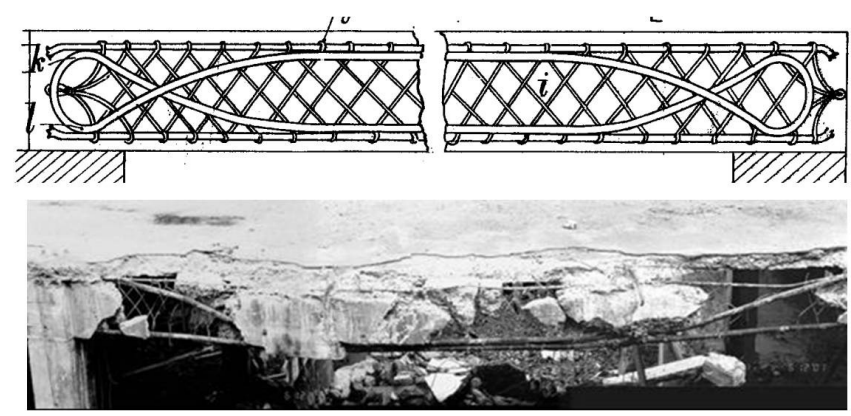

Figura 6. Viga Blanc según patente 29416 (arriba) y en la Alhóndiga Central de Bilbao (abajo). Fuentes: BOPI y los autores

Sin embargo, en lo relativo a los pilares se pusieron de manifiesto dos circunstancias llamativas. La primera es que las barras de los pilares no tenían continuidad entre plantas. En segundo término, se comprobó como los pilares no se encontraban realmente alineados entre plantas, constatando excentricidades apreciables a simple vista.
Tabla 2. Resultados de ensayos de tracción.

\begin{tabular}{|l|c|c|c|c|c|c|}
\hline Propiedad & \multicolumn{6}{|c|}{ Valor } \\
\hline Diámetro (mm) & 5 & 7 & 14,7 & 15 & 24,1 & 48,4 \\
\hline Lím. Elástico (MPa) & 260 & 331 & 260 & 250 & 206 & 198 \\
\hline Tensión de rotura (MPa) & 405 & 433,2 & 342 & 351 & 329 & 295 \\
\hline
\end{tabular}

En la Tabla 2 se incluyen algunos resultados de ensayos sobre probetas de acero del armado. Se observa como todos los valores son claramente superiores a los establecidos según la sistemática de cálculo de Blanc (120 MPa aproximadamente). Destaca la disminución del límite elástico de las muestras a medida que aumenta su diámetro.

Tabla 3. Resistencia estimada del hormigón.

\begin{tabular}{|l|c|}
\hline Elemento estructural & $\begin{array}{c}\text { Resistencia media teórica } \\
\text { (MPa) }\end{array}$ \\
\hline Pilares Sótano & 20,3 \\
\hline Vigas Techo Sótano & 18,4 \\
\hline Pilares Planta Baja & 23,0 \\
\hline Vigas Techo Planta Baja & 11,8 \\
\hline Pilares Planta Primera & 20,3 \\
\hline Vigas Techo Planta Primera & 15,0 \\
\hline Pilares Planta Segunda & 21,6 \\
\hline Vigas Techo Planta Segunda & 8,9 \\
\hline
\end{tabular}

Referido a las resistencias del hormigón, se han obtenido mediante testigos cilíndricos de $75 \mathrm{~mm}$ de diámetro, complementados con ensayos de ultrasonidos en número de 55 valores individuales (Tabla 3), destacándose los bajos resultados en vigas de techo de planta baja y planta segunda (cubierta). No obstante lo cual, los valores medios son muy superiores a los considerados en el sistema Blanc (entre 2,5 y $3 \mathrm{MPa}$ ).

A continuación se llevó a cabo el cálculo de 4 secciones significativas de vigas a flexión en su centro de vano (Tabla 4). Para ello, se ha tenido en cuenta las características del sistema Blanc, adoptando la sobrecarga de diseño del proyecto, de $13,73 \mathrm{kN} / \mathrm{m}^{2}$ (según consta en el archivo antes mencionado, Signatura Bilbao Hacienda 0037/002). Como flector en el centro se adopta el valor $\mathrm{p} \cdot \mathrm{L}^{2} / 10$.

La comparativa se estableció recalculando la armadura según la información original del método y, por otro lado, con propiedades de los materiales deducidas de los ensayos. Es decir, hormigón de 6,2 MPa y acero de límite elástico 200 MPa. La resistencia característica tan baja se justifica por la dispersión de los resultados de los testigos antes ensayados. En todos los casos se ha considerado la posible contribución del forjado a la compresión, sin limitación de ancho efectivo. Las situaciones comparadas fueron las siguientes:

- I: Cálculo de armadura según el método original Blanc (2,45 MPa para el hormigón, y 117,7 MPa para el acero). Toda la seguridad se atribuye a las tensiones admisibles en los materiales. Se considera que la compresión es constante en toda la cabeza comprimida. La fibra neutra se fija arbitrariamente. Acciones sin mayorar.

- II: Cálculo de armadura con propiedades deducidas de ensayos (6,2 MPa para el hormigón, y 200 MPa para acero) 
y coeficientes de seguridad parciales para materiales. Diagrama parábola rectángulo para el hormigón y coeficientes de seguridad de 1,35 para cargas permanentes y 1,5 para sobrecargas.

- III: Cálculo de los momentos últimos con las propiedades actuales, para comparar con las solicitaciones de cálculo.

La viga V1 corresponde a una viga de gran luz (10,20 m.), y es la que presenta un mayor grado de coincidencia en el cálculo de armadura por el método original Blanc. Sin embargo, las vigas $\mathrm{V}_{3}$ y V4 difieren notablemente, contando con un armado inferior al existente. Por otro lado, comparando los momentos últimos con las solicitaciones mayoradas, se aprecia que las tres vigas de menor sección presentan un grado de seguridad no adecuado.

La comprobación ha resultado del todo irregular, atendiendo tanto a criterios actuales como a los criterios originales. Se ha constatado como en flexión el armado sí es capaz de soportar las solicitaciones, basándonos en la fórmula de $\mathrm{p} \cdot \mathrm{L}^{2} / 10$, con unos coeficientes de mayoración de acciones y minoración de la resistencia de los materiales, para las vigas de mayor luz. De forma implícita, la patente contemplaba la absorción de momentos mínimos negativos $\left(\mathrm{p} \cdot \mathrm{L}^{2} / 4 \mathrm{O}\right)$, que son de sobra soportados en cualquier caso por las vigas, debido a las armaduras ascendentes en los extremos.

Referido a la capacidad de carga, y pese a la irregularidad del cálculo, no se tiene constancia de incidencia alguna derivada de su utilización ordinaria. Averiguaciones posteriores de los autores llevan a la conclusión de que la estructura no se ajusta a la sistemática de cálculo Blanc (no se cumple el equilibrio en sección) por lo que se concluye que, en origen, el cálculo podría haberse realizado siguiendo la sistemática de Hennebique/Ribera.

En cuanto a la morfología del armado, en ningún caso puede esperarse plena exactitud con el sistema patentado. Se debe tener en cuenta no solo la adaptación a cada obra sino además que es lógica una evolución en el desarrollo de los armados por cada firma. Otro aspecto a tener en cuenta es que no puede darse por sentado ningún extremo según los estándares actuales de cálculo, proyecto y ejecución. Valga de ejemplo la falta de continuidad de los armados de los pilares entre plantas lo que llevaría, según los criterios actuales de diseño, a la consideración de articulados en la base para cada planta.

\section{CONCLUSIONES}

El hormigón armado tienen su origen en una invención, no en un desarrollo teórico, y por lo tanto la patente busca la exclusividad y, en consecuencia, la rentabilidad económica. Las posibilidades del nuevo material en precio y prestacio- nes tales como la incombustibilidad, facilidad de ejecución, impermeabilidad o monolitismo, hacen que, pese a la desconfianza inicial, vaya ganando mercado a finales del XIX, hasta llegar a un hito clave en Francia, que es la exposición de 1900. Hasta esos momentos, las principales construcciones tienen lugar bajo sistemas patentados, muy numerosos a finales de siglo, si bien se fundamentan más en cuestiones empíricas que en desarrollos teóricos científicamente justificados. En ese sentido, la potencialidad del hormigón, el mercado y la experiencia de las obras ejecutadas se adelanta a su conocimiento.

A principios del siglo XX, las mismas patentes bajo las que se impulsó el hormigón armado, se convierten en un obstáculo para su desarrollo, tanto por su poco carácter científico, como por los derechos económicos y de exclusividad que fomentaban en el sector de la construcción.

Simultáneamente, se producen avances científicos basados en campañas experimentales con el nuevo material, principalmente en Alemania y Francia. Estas circunstancias, sumadas al alarma generada por algunos accidentes de gran trascendencia a nivel europeo, desembocan en la aprobación de las primeras normativas sobre hormigón armado, cuya última finalidad es la de garantizar la seguridad de las estructuras construidas con él.

Las nuevas regulaciones, y la novedosa docencia universitaria, generalizan el conocimiento y aportan criterios técnicos suficientes para abordar el cálculo estructural, sin recurrir a sistemas patentados, cuya formulación queda obsoleta y superada por las normativas de cálculo. Pese a ello, son varias la organizaciones vinculadas a la época de las patentes que prosiguen su actividad una vez superada esta fase, especialmente Wayss \& Freytag y Hennebique, ya que cuentan con capacidades técnicas y comerciales muy notables; la primera aún existe como ingeniería y la segunda prosiguió su actividad hasta 1967.

Sin embargo, al afrontar la rehabilitación de estructuras de esas décadas en las que las patentes tuvieron un gran protagonismo, existe una gran limitación en los conocimientos disponibles acerca de su concepción, cálculo y construcción. Por ello, antes de proceder a la intervención sobre estas estructuras, debe conocerse en profundidad su génesis, si se efectuó bajo patente y las divergencias entre el sistema y la realidad construida en casos similares. Ello permitirá interpretar el comportamiento estructural con mayor conocimiento, y no solamente condicionado por nuestra normativa actual, a todas luces incapaz de tratar estructuras de un pasado tan lejano y complejo.

El estudio presentado de la Alhóndiga de Bilbao, mediante la comparación de los criterios de patente y lo realmente

Tabla 4. Resultados de la comparativo según los tres enfoques en 4 secciones.

\begin{tabular}{|c|c|c|c|c|c|c|}
\hline $\begin{array}{c}\text { Sección mm } \\
\text { (ancho } \times \text { canto visto) }\end{array}$ & $\begin{array}{l}\text { Espesor forjado } \\
(\mathrm{mm})\end{array}$ & $\begin{array}{l}\text { Armadura centro vano } \\
n^{0} \text { y } \varnothing\left(\mathrm{mm}^{2}\right)\end{array}$ & $\begin{array}{c}\mathrm{M}_{\mathrm{f}} \\
\operatorname{Sin} / \operatorname{con} \text { coef. } \\
(\mathrm{kN} \cdot \mathrm{m})\end{array}$ & $\underset{\left(\mathbf{m m}^{2}\right)}{\mathbf{A ~ I ~}}$ & $\underset{\left(\mathbf{m m}^{2}\right)}{\mathbf{A} \text { II }}$ & $\underset{(\mathbf{k N} \cdot \mathbf{m})}{\mathrm{M}_{\mathrm{u}} \text { III }}$ \\
\hline $\mathrm{V} 1(30 \times 60)$ & 120 & $6 \varnothing 50(11781)$ & $781 / 1145$ & 11818 & 9484 & 1185 \\
\hline V2 $(13 \times 45)$ & 100 & $1 \varnothing_{30+1} \varnothing_{32}(1511)$ & $125 / 184$ & 1733 & 1862 & 151 \\
\hline $\mathrm{V}_{3}(10 \times 38)$ & 100 & $2 \varnothing_{28}(1231)$ & $124 / 183$ & 2082 & 1362 & 113 \\
\hline $\mathrm{V}_{4}(13 \times 45)$ & 100 & $2 \varnothing_{30}(1414)$ & 134 / 197 & 1901 & 2014 & 140 \\
\hline
\end{tabular}


dispuesto en obra, permite obtener resultados que pueden condicionar las intervenciones en este tipo de edificios. Una primera impresión podría llevarnos a una conclusión equivocada, acerca de que el edificio no es apto, ya que no cumpliría con los requisitos actuales de seguridad. Sin embargo, la realidad es mucho más compleja que pedir a un edificio de estas características que soporte las cargas según los mismos criterios de cálculo actuales, siendo necesario abordar también otros métodos de estudio como las pruebas de carga, etc.

\section{AGRADECIMIENTOS}

Los autores expresan su agradecimiento a los fondos proporcionados por el Gobierno Vasco en su contrato IT781-13.

\section{REFERENCIAS}

(1) Brown, J. P. (2001). Study in the history of Civil Engineering. London: Newby F.

(2) Simonnet, C. (2009). Hormigón. Historia de un material. San Sebastián: Nerea.

(3) Bosc, J.L., Chauveau, J.M., Clément, J., Degenne, J., Marrey, B., Paulin, M. (2001). Joseph Monier et la naissance du ciment armé. Paris: Éditions du Linteau.

(4) Kierdof, A. (2009). Why Hennebique failed in Germany. Strategies and Obstacles in the introduction of a new construction technology. Cottbus: Brandenburg University of Technology.

(5) Souponitski, S.Z., Sniatkov, S.V., Grigoriev, S.E. (2001). Early reinforced concrete constructions in Russia: specific faults and causes of failure. Engineering Failure Analysis, 8(2): 201-212, doi: http://dx.doi.org/10.1016/S13506307(99)00046-1.

(6) Burgos, A. (2009). Los orígenes del Hormigón Armado en España. Madrid: Ministerio de Fomento, CEDEX-CEHOPU.

(7) Rosell, J., Cárcamo, J. (1994). Los orígenes del hormigón armado y su introducción en Bizkaia. La fábrica Ceres de Bilbao. Bilbao: Colegio Oficial de Aparejadores y A. T. de Vizcaya.

(8) Martín-Nieva, H. (2000). La introducción del hormigón armado en España: las primeras patentes registradas en este país. Madrid: Instituto Juan de Herrera.

(9) Monier, J. (1884). Perfeccionamientos introducidos en las traviesas de ferrocarriles, aplicables a los travesaños para formar los recipientes de todas clases y a las construcciones en general de hierro y cemento (Patente de invención $\mathrm{n}^{\circ}$ 4433). España: Oficina Española de Patentes y Marcas.

(10) Monier, J. (1886). Perfeccionamientos introducidos en las traviesas de ferrocarriles, aplicables a los travesaños para formar los recipientes de todas clases y a las construcciones en general de hierro y cemento, como adición a la patente 6570 expedida el 28 de enero de 1885 (Certificado de adición expediente $n^{0}$ 6156). España: Oficina Española de Patentes y Marcas.

(11) Hennebique, F. (1892). Sistema de combinación especial del metal y del cemento, para la formación de viguetas muy ligeras y de gran resistencia (Patente de invención $\mathrm{n}^{0}$ 13652). España: Oficina Española de Patentes y Marcas.

(12) Hennebique, F. (1898). Mejoras en la construcción de vigas, viguetas y tablones de betún reforzado (Patente de invención $n^{0}$ 22304). España: Oficina Española de Patentes y Marcas.

(13) Delhumeau, G. (1999). L’invention du béton armé Hennebique 189o-1914. Paris: Éditions Norma.

(14) R.O.P. (1897). Cálculo de los pisos de cemento armado, sistema Hennebique. Revista de Obras Públicas, (1116): 122-124.

(15) Compañía Anónima del Hormigón Armado Sestao. (1902). Nota sobre las construcciones de hormigón armado. La Poutre Dalle. Sistema Blanc. Bilbao: Imprenta Antonio Apellaniz.

(16) Ribera, J.E., (1901). El sistema Ribera. El Cemento Armado, (7): 177-179.

(17) Espel, R., Gómez, J., Grima, R., Aguado, A. (2009). La evolución de la construcción del Templo de la Sagrada Familia. Informes de la Construcción, 61(516): 5-20, doi: http://dx.doi.org/10.3989/ic.08.057.

(18) Ribera, J.E. (1902). Mi sistema y mis obras. Madrid: Imprenta Ricardo Rojas.

(19) Ribera, J.E. (1903). Cubiertas para depósitos de agua de hormigón armado sistema Ribera y actas de las pruebas verificadas en Gijón. Madrid: Imprenta Ricardo Rojas.

(20) Ribera, J.E. (1901). Un procedimiento de construcción aplicado a pisos, bóvedas y pilares de hormigón armado (Certificado de adición a la patente 28287, expediente 28861). España: Oficina Española de Patentes y Marcas.

(21) Burgos, A., Saéz-Pérez, M.P., Olmo, J.C. (2012). Carlos Fernández Casado y José Acuña: los primeros puentes de altura estricta. Jaén, 1933-1935. Informes de la Construcción, 64(528): 445-456, doi: http://dx.doi.org/10.3989/ic.11.o82.

(22) Zafra, J. M. (1902). Sistema de piezas de hormigón armado para trabajar por compresión (Patente de invención $\mathrm{n}^{0}$ 29866). España: Oficina Española de Patentes y Marcas.

(23) Marsh, C. F. (1904). Reinforced Concrete. New York: D Van Nostrad Company.

(24) Kurrer, K.E. (2008). The History of the Theory of Structures. From Arch Analysis to Computational Mechanics. Berlin: Ernst \& Sohn.

(25) Burgos-Núñez, A. (2005). El desastre del tercer depósito, cien años después. Revista de Obras Públicas, (3458): 25-48.

(26) Colby, A. L. (1909). Reinforced concrete in Europe. Easton: The Chemical Publishing Company.

(27) France Commission du ciment armé. (1912). The properties and design of reinforced concrete. New York: D Van Nostrad Company.

(28) Marcos, I., San-Mateos, R, Lasarte, N. (2010). Conception and Design of Reinforced Concrete Structures in the Early Twentieth Century: Considerations for Analysis. En 37th IAHS World Congress on Housing. Santander: Universidad de Cantabria. 